\title{
The Method of Jacobi Last Multiplier for Integrating Nonholonomic Systems
}

\author{
Y. ZHANG* \\ College of Civil Engineering, Suzhou University of Science and Technology, Suzhou 215011, China
}

(Received March 30, 2010)

\begin{abstract}
In this paper, a new integral method of nonholonomic dynamical systems is put forward. The differential equations of motion of nonholonomic systems in phase space are established. The definition of the Jacobi last multiplier of the systems is given, and the relation between the Jacobi last multiplier and the first integrals of the systems is discussed. The researches show that the solution of the systems can be found by the last multiplier if the quantity of first integrals of the systems is sufficient. An example is given to illustrate the application of the results.
\end{abstract}

PACS: 45.20.Jj, 02.30.Hq, 02.30.Rz

\section{Introduction}

The integration problem of dynamical equations is a very important problem in analytical mechanics. Although the integration theory of holonomic conservative systems is perfect enough, the integration theory of nonholonomic systems is not quite perfect, and needs to be constantly developed [1-13]. This paper presents a new integration method of nonholonomic systems, i.e., the method of Jacobi last multiplier. The method of Jacobi last multiplier is very important in the problems of differential equations, and some important results have been obtained [14-18]. However, there are few works involving in the application of the Jacobi last multiplier to the nonholonomic systems. In this paper, we further study an application of the method to the nonholonomic systems, establish the relation between the integrals of nonholonomic systems and the Jacobi last multiplier, and take the Appell-Hamel problem as an example to illustrate the application of the results.

\section{Differential equations of motion of nonholonomic systems}

Suppose that the configuration of a mechanical system is determined by $n$ generalized coordinates $q_{s}(s=$ $1, \ldots, n)$. The system is subjected to $g$ ideal nonholonomic constraints of Chetaev's type

$$
f_{\beta}(t, \boldsymbol{q}, \dot{\boldsymbol{q}})=0(\beta=1, \ldots, g) .
$$

The differential equations of motion of the system can be written in the form [11-13]:

\footnotetext{
* e-mail: weidiezh@pub.sz.jsinfo.net
}

$$
\frac{\mathrm{d}}{\mathrm{d} t} \frac{\partial L}{\partial \dot{q}_{s}}-\frac{\partial L}{\partial q_{s}}=Q_{s}^{\prime \prime}+\lambda_{\beta} \frac{\partial f_{\beta}}{\partial \dot{q}_{s}} \quad(s=1, \ldots, n),
$$

where $L$ is the Lagrangian of the system, $Q_{s}^{\prime \prime}-$ nonpotential generalized forces, $\lambda_{\beta}$ - constraint multipliers. Assuming that the system is non-singular, we can seek $\lambda_{\beta}$ as the function of $t, \boldsymbol{q}, \dot{\boldsymbol{q}}$ before integrating the differential equations of motion [11-13].

Introducing the generalized momenta and the Hamiltonian

$$
p_{s}=\frac{\partial L}{\partial \dot{q}_{s}}, \quad H=p_{s} \dot{q}_{s}-L,
$$

then Eqs. (2) can be written in the following form:

$$
\dot{q}_{s}=\frac{\partial H}{\partial p_{s}}, \quad \dot{p}_{s}=-\frac{\partial H}{\partial q_{s}}+\tilde{Q}_{s}^{\prime \prime}+\tilde{\Lambda}_{s} \quad(s=1, \ldots, n),
$$

where

$$
\begin{aligned}
& \tilde{Q}_{s}^{\prime \prime}=\tilde{Q}_{s}^{\prime \prime}(t, \boldsymbol{q}, \boldsymbol{p})=Q_{s}^{\prime \prime}(t, \boldsymbol{q}, \dot{\boldsymbol{q}}(t, \boldsymbol{q}, \boldsymbol{p})), \\
& \tilde{\Lambda}_{s}=\tilde{\Lambda}_{s}(t, \boldsymbol{q}, \boldsymbol{p})=\lambda_{\beta}(t, \boldsymbol{q}, \boldsymbol{p}) \frac{\partial f_{\beta}}{\partial \dot{q}_{s}}(t, \boldsymbol{q}, \boldsymbol{p}) .
\end{aligned}
$$

Let

$$
a^{s}=q_{s}, \quad a^{n+s}=p_{s} \quad(s=1, \ldots, n) .
$$

Then Eqs. (4) can be written in the contravariant algebraic form [12]:

$$
\dot{a}^{\mu}-S^{\mu \nu} \frac{\partial H}{\partial a^{\nu}}=0 \quad(\mu, \nu=1, \ldots, 2 n),
$$

where

$$
S^{\mu \nu}=\omega^{\mu \nu}+T^{\mu \nu}, \quad\left(\omega^{\mu \nu}\right)=\left(\begin{array}{cc}
0_{n \times n} & I_{n \times n} \\
-I_{n \times n} & 0_{n \times n}
\end{array}\right),
$$




$$
\begin{aligned}
& \left(T^{\mu \nu}\right)=\left(\begin{array}{cc}
0_{n \times n} & 0_{n \times n} \\
0_{n \times n} & \left(-\Omega_{k k}\right)_{n \times n}
\end{array}\right), \\
& \tilde{Q}_{s}^{\prime \prime}+\tilde{\Lambda}_{s}=-\Omega_{s k} \frac{\partial H}{\partial p_{k}}, \quad\left(\Omega_{s k}\right)=\left(\begin{array}{ccc}
\Omega_{11} & \ddots & 0 \\
0 & & \Omega_{n n}
\end{array}\right) .
\end{aligned}
$$

The constraints (1) can be expressed as

$$
F_{\beta}=F_{\beta}(t, \boldsymbol{a})=0 \quad(\beta=1, \ldots, g) .
$$

\section{Method of Jacobi last multiplier of nonholonomic systems}

The conception of the last multiplier of a system of differential equations was introduced by Jacobi [1] in 1844. Whittaker gave an application of the last multiplier to a Hamilton system with two degrees of freedom when one integral is known in addition to the integral of energy [1]. Mei and Shang extended the method to a generalized Hamilton system [18]. Now we further apply the method of Jacobi last multiplier to the general nonholonomic systems.

Definition. For a nonholonomic dynamical system under consideration, if there exists some function $M=$ $M(t, \boldsymbol{a})$ satisfying the following condition:

$$
\frac{\overline{\mathrm{d}} M}{\mathrm{~d} t}+M \frac{\partial}{\partial a^{\mu}}\left(S^{\mu \nu} \frac{\partial H}{\partial a^{\nu}}\right)=0,
$$

where

$$
\frac{\overline{\mathrm{d}}}{\mathrm{d} t}=\frac{\partial}{\partial t}+S^{\mu \nu} \frac{\partial H}{\partial a^{\nu}} \frac{\partial}{\partial a^{\mu}}=0,
$$

then the function $M$ is called the Jacobi last multiplier of the system.

Obviously we have

$$
\frac{\partial}{\partial a^{\mu}}\left(\omega^{\mu \nu} \frac{\partial H}{\partial a^{\nu}}\right)=0 .
$$

Therefore the condition (10) is equal to the following equation:

$$
\frac{\overline{\mathrm{d}} M}{\mathrm{~d} t}+M \frac{\partial}{\partial a^{\mu}}\left(T^{\mu \nu} \frac{\partial H}{\partial a^{\nu}}\right)=0 .
$$

Equation (10) or Eq. (13) is called the determining equation of Jacobi last multiplier of the nonholonomic system under consideration.

For the definition above, we have:

Proposition 1. For a nonholonomic dynamical system under consideration, if the following condition is satisfied:

$$
\frac{\partial}{\partial a^{\mu}}\left(T^{\mu \nu} \frac{\partial H}{\partial a^{\nu}}\right)=0 \quad(\mu, \nu=1, \ldots, 2 n),
$$

then $M=1$ is the last multiplier of the system, and all the integrals of the system are its Jacobi last multipliers.

Proposition 2. For a nonholonomic dynamical system under consideration, if $M=M(t, \boldsymbol{a})$ and $N=$
$N(t, \boldsymbol{a})$ are two Jacobi last multipliers of the system, then its quotient $M / N$ is a first integral of the system.

Proof. Since $M=M(t, \boldsymbol{a})$ and $N=N(t, \boldsymbol{a})$ are two Jacobi last multipliers of the nonholonomic system under consideration, according to the definition, we have

$$
\begin{aligned}
& \frac{\partial M}{\partial t}+S^{\mu \nu} \frac{\partial H}{\partial a^{\nu}} \frac{\partial M}{\partial a^{\mu}}=-M \frac{\partial}{\partial a^{\mu}}\left(S^{\mu \nu} \frac{\partial H}{\partial a^{\nu}}\right), \\
& \frac{\partial N}{\partial t}+S^{\mu \nu} \frac{\partial H}{\partial a^{\nu}} \frac{\partial N}{\partial a^{\mu}}=-N \frac{\partial}{\partial a^{\mu}}\left(S^{\mu \nu} \frac{\partial H}{\partial a^{\nu}}\right) .
\end{aligned}
$$

Then we obtain

$$
\begin{aligned}
& \frac{\overline{\mathrm{d}}}{\mathrm{d} t}\left(\frac{M}{N}\right)=\frac{\partial}{\partial t}\left(\frac{M}{N}\right)+S^{\mu \nu} \frac{\partial H}{\partial a^{\nu}} \frac{\partial}{\partial a^{\mu}}\left(\frac{M}{N}\right) \\
& =\frac{1}{N}\left(\frac{\partial M}{\partial t}+S^{\mu \nu} \frac{\partial H}{\partial a^{\nu}} \frac{\partial M}{\partial a^{\mu}}\right) \\
& -\frac{M}{N^{2}}\left(\frac{\partial N}{\partial t}+S^{\mu \nu} \frac{\partial H}{\partial a^{\nu}} \frac{\partial N}{\partial a^{\mu}}\right) \\
& =\frac{1}{N}\left[-M \frac{\partial}{\partial a^{\mu}}\left(S^{\mu \nu} \frac{\partial H}{\partial a^{\nu}}\right)\right] \\
& -\frac{M}{N^{2}}\left[-N \frac{\partial}{\partial a^{\mu}}\left(S^{\mu \nu} \frac{\partial H}{\partial a^{\nu}}\right)\right] \\
& =0 .
\end{aligned}
$$

Therefore, the quotient $M / N$ is a first integral of the system.

Proposition 3. For a nonholonomic dynamical system under consideration, if $(2 n-g-1)$ first integrals of the system are known, i.e.

$$
I_{\sigma}(t, \boldsymbol{a})=C_{\sigma}(\sigma=1, \ldots, \varepsilon ; \varepsilon=2 n-g-1),
$$

then the integral of the system is

$$
\int \frac{M^{*}}{\Delta^{*}}\left[\mathrm{~d} a^{2 n}-\left(S^{2 n, \nu} \frac{\partial H}{\partial a^{\nu}}\right)^{*} \mathrm{~d} t\right]=\text { const },
$$

where $\Delta$ denotes the Jacobian

$$
\Delta=\frac{\partial\left(F_{1}, F_{2}, \ldots, F_{g}, I_{1}, I_{2}, \ldots, I_{\varepsilon}\right)}{\partial\left(a^{1}, a^{2}, \ldots, a^{2 n-1}\right)} .
$$

Here ()$^{*}$ denotes that $a^{\mu}(\mu=1, \ldots, 2 n-1)$ are expressed as functions of $a^{2 n}$ and $t$.

Proof. For the nonholonomic system under consideration, similar to the deduction given by Whittaker in Ref. [1], we can easily obtain

$$
\begin{gathered}
\frac{\partial}{\partial a^{\mu}}\left(S^{\mu \nu} \frac{\partial H}{\partial a^{\nu}}\right)=\Delta\left\{\frac{\partial}{\partial a^{2 n}}\left[\left(S^{2 n, \nu} \frac{\partial H}{\partial a^{\nu}}\right)^{*} / \Delta^{*}\right]\right. \\
\left.+\frac{\partial}{\partial t}\left(\frac{1}{\Delta^{*}}\right)\right\} .
\end{gathered}
$$

Since $M$ is a Jacobi last multiplier of the system, we have 


$$
\frac{1}{M} \frac{\overline{\mathrm{d}} M}{\mathrm{~d} t}+\frac{\partial}{\partial a^{\mu}}\left(S^{\mu \nu} \frac{\partial H}{\partial a^{\nu}}\right)=0 .
$$

From Eq. (18) and (19), we get

$$
\begin{aligned}
& \frac{1}{\Delta M} \frac{\overline{\mathrm{d}} M}{\mathrm{~d} t}+\frac{\partial}{\partial a^{2 n}}\left[\left(S^{2 n, \nu} \frac{\partial H}{\partial a^{\nu}}\right)^{*} / \Delta^{*}\right] \\
& +\frac{\partial}{\partial t}\left(\frac{1}{\Delta^{*}}\right)=0
\end{aligned}
$$

or

$$
\frac{\partial}{\partial a^{2 n}}\left[\left(S^{2 n, \nu} \frac{\partial H}{\partial a^{\nu}}\right)^{*} M^{*} / \Delta^{*}\right]+\frac{\partial}{\partial t}\left(\frac{M^{*}}{\Delta^{*}}\right)=0,
$$

which shows that the expression

$$
\frac{M^{*}}{\Delta^{*}}\left[\mathrm{~d} a^{2 n}-\left(S^{2 n, \nu} \frac{\partial H}{\partial a^{\nu}}\right)^{*} \mathrm{~d} t\right]
$$

is the perfect differential of some function of $a^{2 n}$ and $t$, and this establishes the proposition.

\section{Examples}

In order to illustrate the above results, we study the Appell-Hamel problem [11-13]. The Lagrangian of the problem is

$$
L=\frac{1}{2} m\left(\dot{q}_{1}^{2}+\dot{q}_{2}^{2}+\dot{q}_{3}^{2}\right)-m g q_{3}
$$

and the equation of nonholonomic constraint is

$$
f=\dot{q}_{3}-c\left(\dot{q}_{1}^{2}+\dot{q}_{2}^{2}\right)^{1 / 2}=0 .
$$

The differential equations of motion (2) give

$$
\begin{aligned}
& m \ddot{q}_{1}=-\lambda c \dot{q}_{1}\left(\dot{q}_{1}^{2}+\dot{q}_{2}^{2}\right)^{-1 / 2}, \\
& m \ddot{q}_{2}=-\lambda c \dot{q}_{2}\left(\dot{q}_{1}^{2}+\dot{q}_{2}^{2}\right)^{-1 / 2}, \\
& m \ddot{q}_{3}+m g=\lambda .
\end{aligned}
$$

From Eqs. (23) and (24), we find

$$
\lambda=\frac{m g}{1+c^{2}} .
$$

Also, the generalized momenta and the Hamiltonian are respectively

$$
\begin{aligned}
& p_{1}=\frac{\partial L}{\partial \dot{q}_{1}}=m \dot{q}_{1}, \quad p_{2}=\frac{\partial L}{\partial \dot{q}_{2}}=m \dot{q}_{2}, \\
& p_{3}=\frac{\partial L}{\partial \dot{q}_{3}}=m \dot{q}_{3}, \\
& H=p_{s} \dot{q}_{s}-L=\frac{1}{2 m}\left(p_{1}^{2}+p_{2}^{2}+p_{3}^{2}\right)+m g q_{3} .
\end{aligned}
$$

Then Eqs. (7) yield

where

$$
\dot{a}^{\mu}-S^{\mu \nu} \frac{\partial H}{\partial a^{\nu}}=0 \quad(\mu, \nu=1, \ldots, 6),
$$

$$
H=\frac{1}{2 m}\left[\left(a^{4}\right)^{2}+\left(a^{5}\right)^{2}+\left(a^{6}\right)^{2}\right]+m g a^{3},
$$

$$
\begin{aligned}
& S^{\mu \nu}=\omega^{\mu \nu}+T^{\mu \nu}, \\
& \left(\omega^{\mu \nu}\right)=\left(\begin{array}{cccccc}
0 & 0 & 0 & 1 & 0 & 0 \\
0 & 0 & 0 & 0 & 1 & 0 \\
0 & 0 & 0 & 0 & 0 & 1 \\
-1 & 0 & 0 & 0 & 0 & 0 \\
0 & -1 & 0 & 0 & 0 & 0 \\
0 & 0 & -1 & 0 & 0 & 0
\end{array}\right), \\
& \left(T^{\mu \nu}\right)=\left(\begin{array}{cccccc}
0 & 0 & 0 & 0 & 0 & 0 \\
0 & 0 & 0 & 0 & 0 & 0 \\
0 & 0 & 0 & 0 & 0 & 0 \\
0 & 0 & 0 & -\Omega_{11} & 0 & 0 \\
0 & 0 & 0 & 0 & -\Omega_{22} & 0 \\
0 & 0 & 0 & 0 & 0 & -\Omega_{33}
\end{array}\right), \\
& \Omega_{11}=\frac{m^{2} g c}{1+c^{2}}\left[\left(a^{4}\right)^{2}+\left(a^{5}\right)^{2}\right]^{-1 / 2}, \\
& \Omega_{22}=\frac{m^{2} g c}{1+c^{2}}\left[\left(a^{4}\right)^{2}+\left(a^{5}\right)^{2}\right]^{-1 / 2}, \\
& \Omega_{33}=-\frac{m^{2} g}{1+c^{2}}\left(a^{6}\right)^{-1} .
\end{aligned}
$$

The equation of constraint (23) can be written as

$$
F=a^{6}-c\left[\left(a^{4}\right)^{2}+\left(a^{5}\right)^{2}\right]^{1 / 2}=0 .
$$

The determining equation of the Jacobi last multiplier gives

$$
\frac{\overline{\mathrm{d}} M}{\mathrm{~d} t}-M \frac{m g c}{1+c^{2}}\left[\left(a^{4}\right)^{2}+\left(a^{5}\right)^{2}\right]^{-1 / 2}=0 .
$$

Equation (31) has a solution

$$
M=c\left[\left(a^{4}\right)^{2}+\left(a^{5}\right)^{2}\right]^{-1 / 2} .
$$

In this problem, the system has the following integrals [12]:

$$
\begin{aligned}
& I_{1}=\frac{1}{2 m}\left[\left(a^{4}\right)^{2}+\left(a^{5}\right)^{2}+\left(a^{6}\right)^{2}\right]+m g a^{3}=C_{1}, \\
& I_{2}=\frac{1}{m} a^{5} a^{6}+m g a^{2}=C_{2}, \\
& I_{3}=\frac{1}{m} a^{4} a^{6}+m g a^{1}=C_{3}, \\
& I_{4}=\frac{a^{4}}{a^{5}}=C_{4} .
\end{aligned}
$$

From Eq. (30) and the first integrals (33)-(36), we have

$$
\begin{aligned}
& a^{1}=\frac{1}{m g}\left[C_{3}-\frac{C_{4}}{m c\left(1+C_{4}^{2}\right)}\left(a^{6}\right)^{2}\right], \\
& a^{2}=\frac{1}{m g}\left[C_{2}-\frac{1}{m c\left(1+C_{4}^{2}\right)}\left(a^{6}\right)^{2}\right],
\end{aligned}
$$




$$
\begin{aligned}
a^{3} & =\frac{1}{m g}\left[C_{1}-\frac{1+c^{2}}{2 m c^{2}}\left(a^{6}\right)^{2}\right], \quad a^{4}=\frac{C_{4}}{c\left(1+C_{4}^{2}\right)} a^{6}, \\
a^{5} & =\frac{1}{c\left(1+C_{4}^{2}\right)} a^{6} .
\end{aligned}
$$

Making some deriving work, we obtain

$$
\begin{aligned}
\Delta & =\frac{\partial\left(F, I_{1}, I_{2}, I_{3}, I_{4}\right)}{\partial\left(a^{1}, a^{2}, a^{3}, a^{4}, a^{5}\right)} \\
& =c m^{3} g^{3}\left[\left(a^{4}\right)^{2}+\left(a^{5}\right)^{2}\right]^{1 / 2}\left(a^{5}\right)^{-2}, \\
\Delta^{*} & =c^{2} m^{3} g^{3}\left(1+C_{4}^{2}\right)^{2}\left(a^{6}\right)^{-1} .
\end{aligned}
$$

Expression (16) gives the following integral:

$$
\int \frac{c^{2}\left(a^{6}\right)^{-1}}{c^{2} m^{3} g^{3}\left(1+C_{4}^{2}\right)^{2}\left(a^{6}\right)^{-1}}\left(\mathrm{~d} a^{6}+\frac{m g c^{2}}{1+c^{2}} \mathrm{~d} t\right)=C_{5} .
$$

After integrating, we obtain

$$
a^{6}=-\frac{m g c^{2}}{1+c^{2}} t+C_{5} m^{3} g^{3}\left(1+C_{4}^{2}\right)^{2} .
$$

Formulae (37) and (41) give the solution of the AppellHamel problem, and it contains five arbitrary constants.

\section{Conclusions}

This paper presents a new method, i.e., the method of the Jacobi last multiplier, to integrate the dynamical equations of nonholonomic systems. In principle, the method of the Jacobi last multiplier is applicable for solving the integration problem of the equations of motion of general nonholonomic systems, and it has no restrictions such as those in the Hamilton-Jacobi method. The main difficulty of this method lies in: one is that a sufficient number of independent first integrals must be known in advance; the other is to seek the solution of the determining Eq. (13). However, as long as $2 n-g-1$ first integrals are known and the Jacobi last multiplier of the system might be found, one can obtain the solution of the nonholonomic system.

\section{Acknowledgments}

Project supported by the National Natural Science Foundation of China (grant No. 10972151).

\section{References}

[1] E.T. Whittaker, A Treatise on the Analytical Dynamics of Particles and Rigid Bodies, 4th ed., Cambridge University Press, Cambridge 1952.

[2] F.X. Mei, D. Liu, Y. Luo, Advanced Analytical Mechanics, Beijing Institute of Technology Press, Beijing 1991 (in Chinese).

[3] F.X. Mei, H.B. Wu, H.P. Zhu, Chin. Sci. Bull. 40, $1442(1995)$

[4] F.X. Mei, Int. J. Non-Linear Mech. 35, 229 (2000).

[5] F.X. Mei, Appl. Mech. Rev. Am. Soc. Mech. Eng. 53, $283(2000)$.

[6] Y.X. Guo, M. Shang, S.K. Luo, F.X. Mei, Int. J. Theor. Phys. 40, 1197 (2001).

[7] H.B. Zhang, L.Q. Chen, Mech. Res. Commun. 32, $628(2005)$.

[8] F.X. Mei, H.B. Wu, Phys. Lett. A 372, 2141 (2008).

[9] J.L. Fu, S. Jiménez, Y.F. Tang, L. Vázquez, Phys. Lett. A 372, 1555 (2008).

[10] J.L. Fu, B.Y. Chen, L.Q. Chen, Phys. Lett. A $\mathbf{3 7 3}$ 409 (2009).

[11] Ju. I. Neimark, N.A. Fufaev, Dynamics of Nonholonomic Systems, Nauka, Moscow 1967 (in Russian).

[12] F.X. Mei, Applications of Lie Groups and Lie Algebras to Constrained Mechanical Systems, Science Press, Beijing 1999 (in Chinese).

[13] J.G. Papastavridis, Analytical Mechanics, Oxford University Press, New York 2002.

[14] M.C. Nucci, P.G.L. Leach, J. Phys. A, Math. Gen. 37, 7743 (2004).

[15] M.C. Nucci, J. Nonlinear Math. Phys. 12, 284 (2005).

[16] M.C. Nucci, P.G.L. Leach, J. Math. Phys. 48, 013514 (2007).

[17] M.C. Nucci, P.G.L. Leach, J. Math. Phys. 49, 073517 (2008).

[18] F.X. Mei, M. Shang, Chin. Phys. Lett. 25, 3837 (2008). 